\title{
Comparative Study of Chain Mobility Models using Reactive Routing protocols
}

\author{
Anil Bhasin \\ Dept. of CS, HMV Jalandhar. INDIA
}

\author{
Dinesh Kumar \\ Dept. of IT, DAVIET Jalandhar.INDIA
}

\begin{abstract}
A mobile ad hoc network is a collection of wireless mobile nodes that communicate with each other without centralized control or established infrastructure. The mobility model should represent the realistic behavior of each mobile node in the ad hoc network as routing protocols performances vary widely across the different mobility models. Since not many mobile ad hoc networks are currently deployed, research in this area is mostly simulation based. In this paper, we focus on the impact of Chain mobility models on the performance of Reactive routing protocols. With the help of NS2 simulator we investigated various simulation scenarios by varying number of mobile nodes.
\end{abstract}

Keyword

Mobile Adhoc Networks, Mobility Models, Routing Protocols, Performance parameters.

\section{INTRODUCTION}

Mobile Ad-Hoc Network is a self-configuring network of mobile nodes connected by wireless. It forms an arbitrary topology without any use of existing infrastructure [1]. To communicate with each other nodes of the network have radio range. Nodes outside this range rely on their neighbor mobile nodes to forward packets. Mobile nodes are free to join or leave the network at any point of time, resulting in a highly dynamic network environment. Every mobile node behaves either a host or a router at instant of time.

Because the nodes are free to move randomly, the topology of network changes rapidly and may be unpredictable. These scenarios make the traditional protocol not suitable for Ad hock network. Ad hock networks have characteristics like Dynamic topologies, Bandwidth constrained, Variable capacity links, battery constrained operation, security [2]. Therefore the routing protocols used in ordinary wired networks can not perform well for this level of dynamic environment.

What pattern a node follows will have a significant impact on the network performance. The mobility model has been widely used for evaluating the performance of relevant protocols or algorithms in traditional Ad-hoc networks [3]. Various mobility models have been proposed in the recent past to understand their behavior.

Among these models Random Walk Mobility Model is simplest. In this model mobile nodes moves with random directions and speeds. Feature of pause time is added in Random Waypoint Mobility Model when ever there is change in destination and speed. Random Direction Mobility Model forces mobile nodes to travel to the edge of the simulation area before changing direction and speed. GaussMarkov Mobility Model uses one tuning parameter to vary the degree of randomness in the mobility pattern. A
Probabilistic Version of the Random Walk Mobility Model utilizes a set of probabilities to determine the next position of an MN. City Section Mobility Model represents a simulation area of streets within a city [4]. The Manhattan model [7] is used to emulate the movement pattern of mobile nodes on streets environment. Hierarchical routing based Reference Point Group Mobility model has a logic center (group leader) to determine the movement of the whole group. The Chain model permits to specify a few models including Random Waypoint, Manhattan, Reference Point Group Mobility model [5].

Routing in such networks is a challenge for sending data from one node to other node. Ad hock network uses multi-hop routing instead of a static network infrastructure to provide network connectivity. Several routing protocols have been proposed in literature for mobile Ad Hoc networks [6]. There are various models based on different criteria for designing and classifying routing protocols for such networks. For example, what routing information is exchanged, when and how the routing information is exchanged, when and how routes are computed etc. Routing protocols for Mobile ad hoc networks has been classified into two main categories [8]. Proactive (table-driven routing) and Reactive (on-demand routing).Table-driven routing protocols attempt to maintain consistent, up-to-date routing information from each node to every other node in the network. Destination Sequenced Distance Vector, Optimised Link State Routing are example of this type. On-demand routing protocols were designed to reduce the overheads in Table-Driven protocols by maintaining information for active routes only. On-Demand routing protocols are classified into source routing and hop by hop routing. In source routing (e.g. Dynamic Source Routing) protocol each data packets carry the complete path from source to destination. Therefore, each intermediate node forwards these packets according to the information in the header of each packet. In hop-by-hop routing protocol (e.g. Adhoc on-Demand Distance Vector protocol) each data packet only carries the destination address and the next hop address. Therefore, each intermediate node in the path to the destination uses its routing table to forward each data packet towards the destination. The routes are adaptable to the dynamically changing environment of MANETs. Each node updates its routing table when they receive fresh topology information from its neighbor mobile modes and forward this data packets over the network for new and better routes. Using this information means that fewer route recalculations are required during data transmission.

We have used the DSR and AODV, both reactive routing protocols against two different chain mobility patterns. We tried to establish the comparative study of their performance on the basis of performance metrics like throughput, packet 
delivery fraction and end to end delay through simulations. The rest of the paper is organized as follows: Section 2 describes the traditional mobility models and Reactive routing protocols. Section 3 describes the simulation scenario, Section 4 describes results and Section 5 concludes the paper.

\section{RELATED WORK}

Wireless interfaces of the mobile nodes allow communication between them with one and another. Due to mobility of nodes, limited radio range, failure of mobile nodes and large area, wireless networks are often vulnerable to network partitioning. Unfortunately, most existing ad hoc routing protocols will fail to deliver messages under these circumstances since no route to the destination exists. Zhao etal [9] propose the Message Ferrying scheme that provides efficient data delivery in disconnected wireless ad hoc networks. In the message ferrying scheme, nodes move proactively to send or receive messages. By introducing non-randomness in a node's proactive movement and exploiting such nonrandomness to deliver messages, the message ferrying scheme improves data delivery performance in a disconnected network.

According to Bettstetter etel [10] the Random Waypoint Mobility Model includes pause times between changes in direction and/or speed. Mobile node begins by staying in one location for a certain period of time (i.e., a pause time). Once this time expires, the mobile node chooses a random destination in the simulation area and a speed that is uniformly distributed between [minspeed, maxspeed]. The mobile node then travels toward the newly chosen destination at the selected speed. Upon arrival, the MN pauses for a specified time period before starting the process again. The movement pattern of an mobile nodes using the Random Waypoint Mobility Model is similar to the Random Walk Mobility Model if pause time is zero and [minspeed, maxspeed $]=$ [speedmin, speedmax $]$.

By Sadagopan etal [11] Reference Point Group Mobility model has a special mobile node known as the logical center. The motion of this mobile node defines the entire group's features like location, speed, direction, acceleration, etc. Thus, the group trajectory is determined by providing a path for the center. Generally nodes are uniformly distributed within the geographic range of a group. Each node is assigned a reference point which follows the group movement. This reference point allows independent random motion behavior for each node, in addition to the group motion. In fig.(1) node 1 and 2 are group leaders.

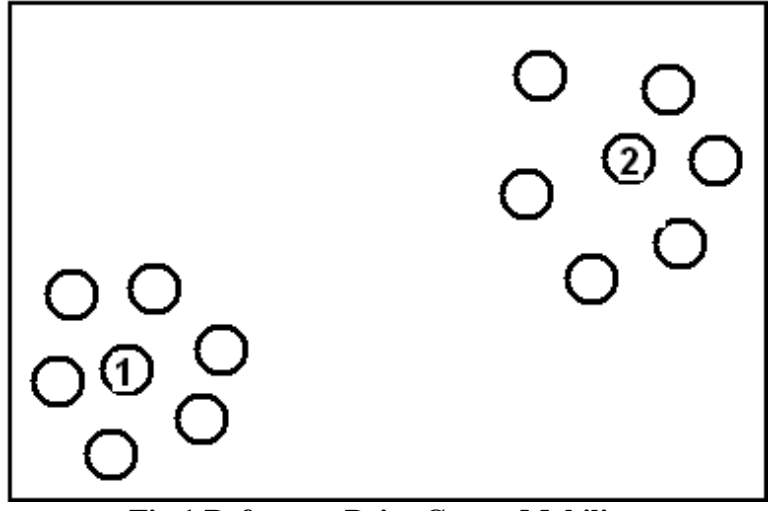

Fig.1 Reference Point Group Mobility

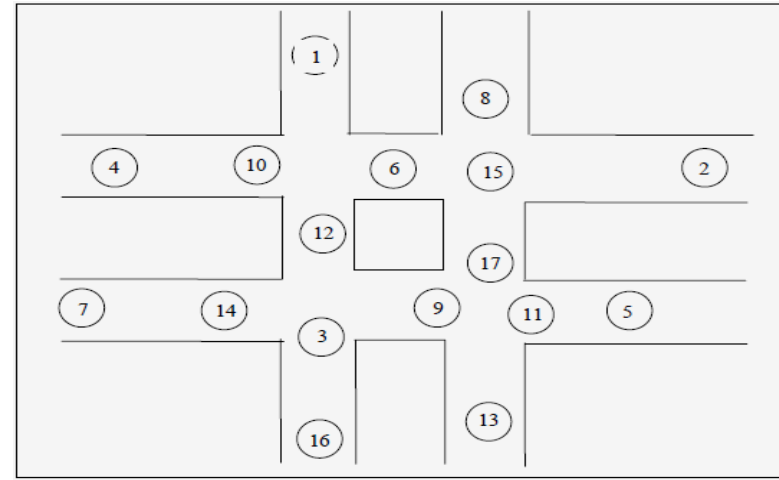

Fig. 2 Manhattan Mobility Model

Manhattan Mobility Model is used to emulate the movement pattern of mobile nodes on streets [12] (figure 2). It can be useful in modeling movement in an urban area. In this network maps are used. Maps contain a number of horizontal and vertical streets. The mobile nodes are restricted to move along horizontal and vertical streets on the map. At an intersection of a horizontal and a vertical street, the mobile node can move left, right, straight with certain probability. The speed of a mobile node at sometime is dependent on its previous time speed and on the speed of the front node in the same direction.

\section{SIMULATION SCENARIO}

We performed simulation to compare and bring in performance behavior of DSR and AODV routing protocols against the two different chain mobility models. We assumed the nodes of network to vary from 5 to 20 to understand the quality of service of the two protocols. The parameters for the simulation are described in table 1.

\section{Table 1}

\begin{tabular}{|l|l|}
\hline Parameter & Value \\
\hline Simulator & NS-2 (2.34) \\
\hline Routing Protocols & DSR, AODV \\
\hline Send Rate & $0.125 \mathrm{sec}$ \\
\hline Simulation Area & $500 * 500$ \\
\hline Traffic Type & CBR (UDP) \\
\hline Maximum number of Nodes & 25 \\
\hline Node Movement Model & $\begin{array}{l}\text { Chain Campus, } \\
\text { Chaintest Random }\end{array}$ \\
\hline
\end{tabular}

\section{RESULT INVESTIGATION}

The source routing protocols (e.g. DSR) have a longer delay because each data packets carry the complete path from source to destination. This slows down the transmission of packets and route discovery takes more time as every intermediate node tries to extract information from routing table before forwarding the reply. In hop by hop routing protocols (e.g. AODV) each data packet carries the destination address and the next hop address only. An attempt has made to evaluate the performance of (DSR and AODV ) reactive routing by varying the Mobility pattern and Number of traffic sources. 
Throughput is the time taken by the total size of useful packets that are received at all the destination nodes. The Throughput of the reactive routing protocols is same for Chaintest Random and Chain Campus mobility models. DSR data packets contains the path from source to destination so it may refer out dated router table but in AODV data packet contains the destination address and the next hop address and it may refer latest router table. As the number of nodes is small both reactive routing protocols performance is same. This is shown in Fig 3 (a) \& Fig 3 (b) using Table 2(a) \& Table 2(b)
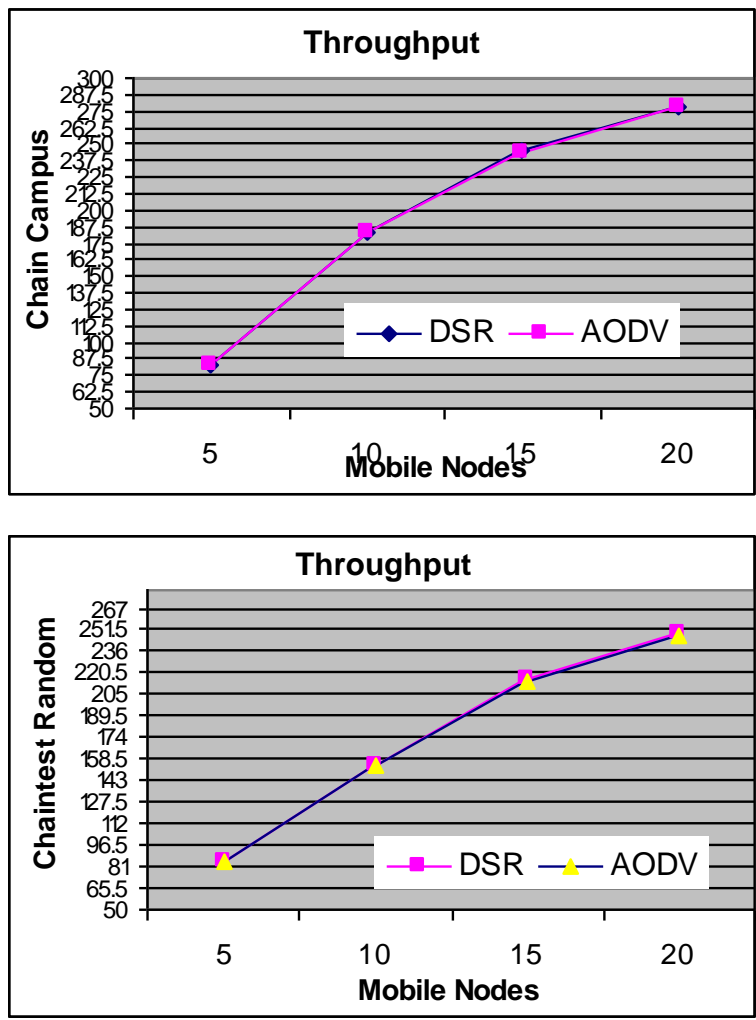

Fig. 3(a, b) Throughput (in Kbps) of Chain mobility models using Reactive routing protocols

Table 2(a) Throughput for Chain Campus

\begin{tabular}{|c|c|c|}
\hline Node & Throughput-DSR & Throughput-AODV \\
\hline 5 & 83.1 & 83.52 \\
\hline 10 & 182.7 & 183.28 \\
\hline 15 & 246.1 & 244 \\
\hline 20 & 278.83 & 278.89 \\
\hline
\end{tabular}

Table 2(b) Throughput for Chaintest Random

\begin{tabular}{|c|c|c|}
\hline Node & Throughput-DSR & Throughput-AODV \\
\hline 5 & 83.84 & 83.87 \\
\hline 10 & 153.1 & 152.99 \\
\hline 15 & 215.48 & 214.52 \\
\hline 20 & 248.57 & 247.34 \\
\hline
\end{tabular}

Packet Delivery Fraction (PDF) is the ratio of the data packets delivered to the destinations to those generated by the sources. We used the relation PDF $=$ Total Packets Delivered to destination / Total Packets Generated. The Packet Delivery Fraction for DSR and AODV routing protocols is same for Chaintest Random mobility models. Results show that packet delivery fraction of AODV is higher than DSR with smaller number of nodes in chain campus mobility model. This is because DSR uses caching aggressively and lack of any mechanism to determine the novelty of routes or expiry of out dated routes when multiple choices are available as shown in Fig 5(a) \& Fig. 5 (b).
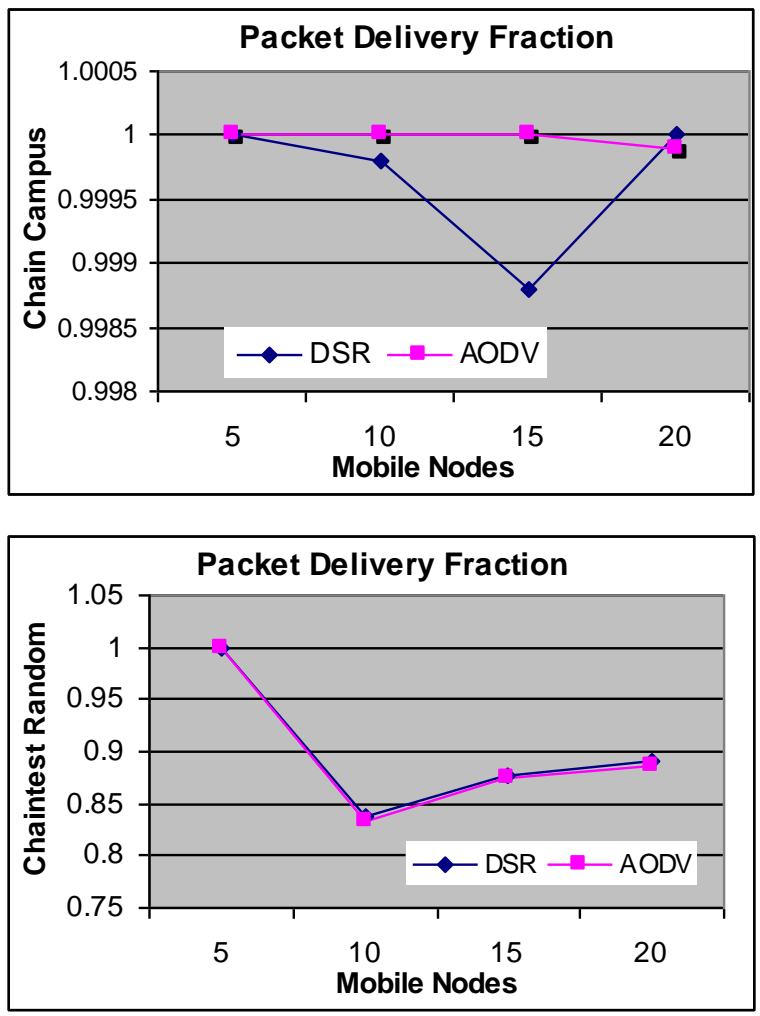

Fig. 4(a, b) Packet Delivery Fraction of Chain mobility models using Reactive routing protocols

DSR generates less routing load than AODV so as the number of mobile nodes increases its performance comes at par or it may be outperform AODV in chain campus mobility model.

End to End Delay (Quality of Service) indicates the time a packet takes to travel from the source to the destination. This is the sum of delays from buffering during route discovery latency, queuing at the interface queue, retransmission delays and transportation times of data packets. From the results as shown in fig5(a) \& fig5(b) the average packet delay time increase for increase in number of nodes waiting for the routing protocols to find valid route to the destination. Delay time is also affected by route discovery. The End to End Delay in Chain Campus mobility model show that AODV has better performance as compare to DSR, but in case of Chaintest Random mobility model the DSR outperforms than AODV. 

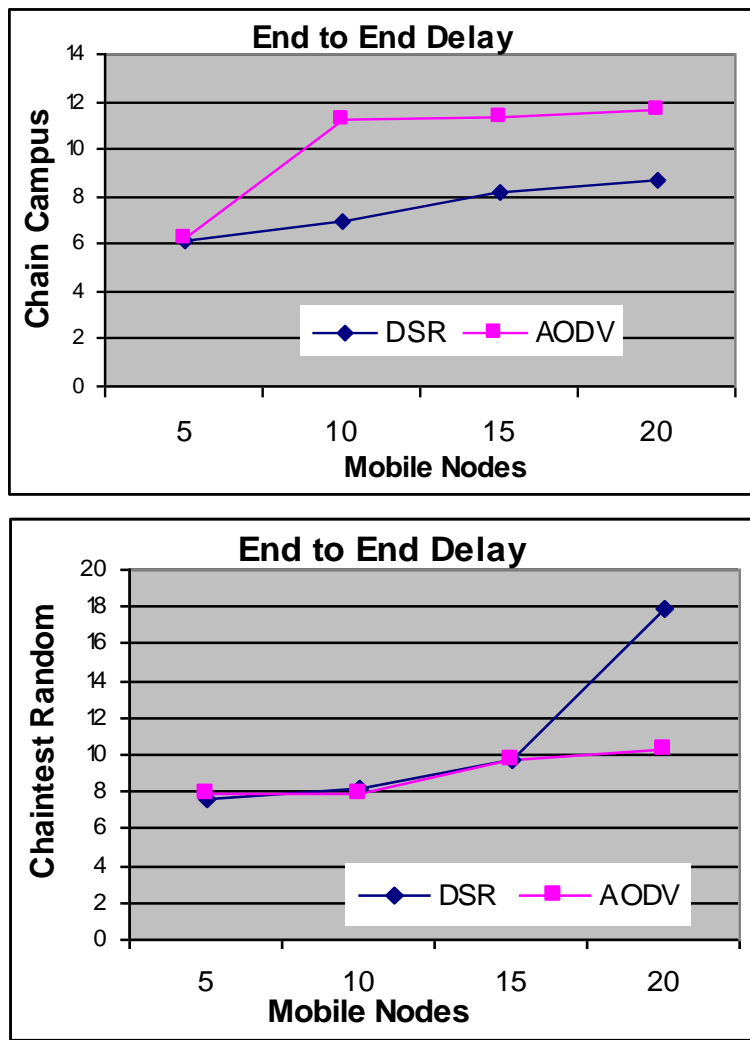

Fig. 5(a,b) End to End Delay (in msec) of Chain mobility models using Reactive routing protocols

\section{CONCLUSION}

The mobile nodes in the Adhoc network move unscientifically. We are not sure about the position of the mobile nodes, we are not certain about the location of link node. These systems generally require long-range radio or interrupt nodes' mobility patterns. We have compared the performance of chain Mobility models on reactive routing protocols using three metrics. Throughput the quantity parameter metrics of routing protocols, increases as the number of mobile nodes increases in both chain models. In Chain campus mobility model the Packet Delivery Fraction metric for Adhoc On-Demand Distance Vector protocol remains consistent while Dynamic Source Routing protocol varies slightly. With Chain campus mobility model End to End Delay metrics Dynamic Source Routing protocol performs better than Adhoc On-Demand Distance Vector protocol. In case of Chaintest random mobility model Adhoc On-Demand Distance Vector protocol performs better than Dynamic Source Routing protocol as the number of connected mobile nodes increases.

\section{REFRENCES}

[1] Liu Tie-yuan, CHANG Lian, Gu Tian-long "Analyzing the Impact of Entity Mobility Models on the Performance of Routing Protocols in the MANET" Third International Conference on Genetic and Evolutionary Computing, pp.56-59@ 2009 IEEE

[2] Anuj K. Gupta, Dr. Harsh Sadawarti, Dr. Anil K. Verma "Performance analysis of AODV, DSR \& TORA Routing Protocols" International Journal of Engineering and Technology, Vol.2, No.2, April 2010

[3] Yang Huan, Jiang Hong, Liu Lei "Performance Analysis of Mobility Models in Sparse Ad-hoc Networks," Proceedings of the 27th Chinese Control Conference, Kunming,Yunnan, China, pp. 216-220, July 16-18, 2008

[4] Tracy Camp, Jeff Boleng, Vanessa Davies "A Survey of Mobility Models for Ad Hoc Network Research" Wireless Communication \& Mobile Computing (WCMC): Special issue on Mobile Ad Hoc Networking: Research, Trends and Applications,vol.2, no. 5, pp. 483502,2002

[5] www.net.cs.unibonn.de/fileadmin/ag/martini/.../Bonn Motion_Docu.pdf

[6] Sapna S. Kaushik \& P.R.Deshmukh "comparison of effectiveness of aodv, dsdv and dsr routing protocols in mobile ad hoc networks" International Journal of Information Technology and Knowledge Management, , Vol. 2, No. 2, pp. 499-502, July-December 2009

[7] Hong x, Gerla m, Pei g, Chiang c c. "A group mobility model for ad hoc wireless networks" In Proc. ACM/IEEE MSWiM, 1999.

[8] Narendra Singh Yadav, R.P.Yadav, "Performance Comparison and Analysis of Table- Driven and OnDemand Routing Protocols for Mobile Ad-hoc Networks" International Journal of Information Technology Vol. 4 No.2, 2002

[9] Wenrui Zhao; Ammar, M.H.; "Message ferrying: proactive routing in highly-partitioned wireless ad hoc networks" FTDCS '03 Proceedings of the The Ninth IEEE Workshop on Future Trends of Distributed Computing Systems, pp 308-314, 2003

[10] Bettstetter C, Recta G, Santi P. "The node distribution of the Random Waypoint mobility model for wireless ad hoc net works" IEEE Trans. Mobile Computing, pp: 257-269,2003

[11] Bai F, Sadagopan N, Helmy “A. Important: a framework to systematically analyze the impact of mobility on performance of routing protocols for ad hoc networks" In Proc. IEEE Information Communications Conference INFOCOM 2003.

[12] Mousavi, S.M. Rabiee, H.R. Moshref, "MobiSim: A Framework for Simulation of Mobility Models in Mobile Ad-Hoc Networks" IEEE International conference WiMOB 2007. 